\title{
Assessment of molecularly imprinted polymers (MIPs) in the preconcentration of disperse red 73 dye prior to photoelectrocatalytic treatment
}

\author{
Jefferson Honorio Franco ${ }^{1}$ • Alejandra Ben Aissa ${ }^{2}$ - Guilherme Garcia Bessegato ${ }^{1}$. \\ Laura Martinez Fajardo ${ }^{2}$ - Maria Valnice Boldrin Zanoni ${ }^{1}$ - María Isabel Pividori ${ }^{2}$ • \\ Maria Del Pilar Taboada Sotomayor ${ }^{1}$
}

Received: 16 August 2016 / Accepted: 14 November 2016/Published online: 8 December 2016

(C) Springer-Verlag Berlin Heidelberg 2016

\begin{abstract}
Magnetic molecularly imprinted polymers (MMIPs) have become a research hotspot due to their two important characteristics: target recognition and magnetic separation. This paper presents the preparation, characterization, and optimization of an MMIP for the preconcentration of disperse red 73 dye (DR73) and its subsequent efficient degradation by photoelectrocatalytic treatment. The MMIPs were characterized by scanning electron microscopy (SEM), which revealed homogeneous distribution of the particles. Excellent encapsulation of magnetite was confirmed by transmission electron microscopy (TEM). A study of dye binding showed that the dye was retained more selectively in the MIP, compared to the NIP. The release of DR73 from the imprinted polymers into methanol and acetic acid was analyzed by UV-Vis spectrophotometry. The extracts showed higher absorbance values for MMIP, compared to MNIP, confirming greater adsorption of dye in the MMIP material. The extracts were then subjected to photoelectrocatalytic treatment. LC-MS/ MS analysis following this treatment showed that the dye was almost completely degraded. Hence, the combination of MMIP extraction and photoelectrocatalysis offers an alternative way of selectively removing an organic contaminant, prior to proceeding with its complete degradation.
\end{abstract}

Responsible editor: Vítor Pais Vilar

Electronic supplementary material The online version of this article (doi:10.1007/s11356-016-8116-9) contains supplementary material, which is available to authorized users.

Maria Del Pilar Taboada Sotomayor mpilar@iq.unesp.br; mpilarts@hotmail.com

1 Department of Analytical Chemistry, Institute of Chemistry, São Paulo State University (UNESP), São Paulo, Brazil

2 Group of Sensors and Biosensors, Autonomous University of Barcelona, Barcelona, Spain
Keywords Molecularly imprinted polymers · Textile dye degradation $\cdot$ Photoelectrocatalytic oxidation $\cdot$ Wastewater treatment $\cdot$ Adsorption $\cdot$ Advanced oxidation process

\section{Introduction}

Textile industries produce large amounts of wastewater containing organic and inorganic compounds. In a dyeing process, not all the dye is fixed to the fibers, with around 10 $15 \%$ being lost in the form of a highly colored effluent (Jin et al. 2007; Ghaly et al. 2014). Around 280,000 t of textile dyes are released from these industries worldwide every year (Jin et al. 2007; Saratale et al. 2011). Among the various classes of dyes, azo compounds represent about $70 \%$ of the total, making them the most widely used synthetic dyes. By virtue of their complex chemical structures, dyes are resistant to fading due to exposure to light, water, and a wide range of chemicals, so they are difficult to decolorize (Robinson et al. 2001). The direct discharge of these effluents can lead to the formation of toxic aromatic amines under anaerobic conditions. In addition to their visual effects and adverse impacts in terms of chemical oxygen demand, many synthetic dyes are toxic, mutagenic, and carcinogenic (Jin et al. 2007).

Many of these synthetic dye residues are unable to be treated successfully using conventional methods such as municipal aerobic treatment systems (Arslan et al. 1999; Robinson et al. 2001). This, together with stricter regulations concerning effluent discharges, has led to research efforts to identify treatment methods that are more efficient, and a wide range of decontamination techniques have been proposed (Panizza and Cerisola 2008).

Adsorption techniques have attracted attention due to their efficiency in the removal of pollutants that are resistant to 
elimination by conventional methods. Activated carbon is the most popular adsorbent used for the removal of pollutants from wastewater (Yagub et al. 2014). Other low-cost adsorbents include agricultural solid wastes such as bark, sawdust, and chitosan (Salleh et al. 2011); industrial byproducts including metal hydroxide sludge, fly ash, red mud, biosolids, and slurry waste (Ahmaruzzaman 2011); inorganic materials such as clay minerals, siliceous materials, and zeolites (Yagub et al. 2014); and biosorbents and microbial biomass (Sanghi and Bhattacharya 2002). Among the adsorption techniques that have been studied for the removal of compounds in effluents, the use of molecularly imprinted polymers (MIPs) has been suggested as a novel, promising, and innovative technique for the removal of dyes from wastewaters (Murray and Örmeci 2012; Huang et al. 2014).

MIPs are synthetic materials capable of binding with target analytes (Lavignac et al. 2004). The origin of molecular imprinting of inorganic and organic polymers goes back to Pauling's in vitro production of antibodies and Fischer's lock and key principle, which was described for the first time by Wulff and collaborators (Wulff and Sarhan 1972; Wulff et al. 1997). MIPs are synthetic crosslinked polymers that possess specific cavities designed for a target molecule (the template) (Shea and Sasaki 1991; Vlatakis et al. 1993). They have the ability to recognize and selectively bind to the target analyte, in the same way as an antibody, and present a number of advantages including easy preparation and high thermal and chemical stability (Svenson and Nicholls 2001; Jiang et al. 2006).

Typically, the generation of stable high-affinity MIPs requires the following: (i) one or more functional monomers capable of forming stable complexes with the template molecule; (ii) a high degree of cross-linking to preserve the templated sites; and (iii) the use of an aprotic apolar solvent as the porogen (Dirion et al. 2003).

Functional monomers are responsible for the binding interactions at the imprinted sites, while non-covalent molecular imprinting employs an excess of monomers, relative to the number of moles of template, in order to favor formation of the template and the functional monomer assemblies (Tamayo et al. 2003).

It should be noted that like other adsorption methods, MIPs can only remove the contaminant from water, without destroying it (Murray and Örmeci 2012; Huang et al. 2014). Hence, as MIPs can selectively adsorb an organic compound, it is possible to remove a specific target molecule from a contaminated wastewater and subsequently release it for efficient destruction using an advanced oxidation process.

Photoelectrocatalytic elimination of organic pollutants is an effective advanced oxidation process that provides improved photocatalytic efficiency by applying an electrochemical potential. The choice of a suitable photocatalyst is crucially important in photoelectrocata lysis. $\mathrm{TiO}_{2}$ is the most widely used photocatalyst, due to its availability, low cost, and non-toxicity, as well as the fact that it has sufficient valence band potential for the oxidation of $\mathrm{H}_{2} \mathrm{O} / \mathrm{OH}^{-}$to hydroxyl radicals $\left({ }^{\circ} \mathrm{OH}\right)$ (Bessegato et al. 2015). $\mathrm{TiO}_{2}$ nanotube array electrodes have been demonstrated to be the most appropriate morphology, because their precise structural orientation provides excellent electron percolation pathways for vectorial charge transfer, which reduces electron/hole recombination (Grimes and Mor 2009; Liu et al. 2012).

The increasingly stringent legislation concerning the removal of dyes from industrial effluents has focused research efforts on developing new treatment techniques suitable for use by the textile industry. Photoelectrocatalytic processes offer effective ways to reduce the concentrations of textile dyes (Carneiro et al. 2009; Paschoal et al. 2009). They can be used to treat aqueous textile industry effluents (Cardoso et al. 2015), as well as for treating wastewater effluents reaching public sewage treatment plants, where the dye is usually diluted and found at low concentration (Xie and Li 2006).

This work describes the synthesis and characterization of magnetic molecularly imprinted polymers (MMIPs) selective toward disperse red 73 (DR73, Fig. 1) and suitable for removal of the dye from aqueous media. Evaluation was then made of extraction of the dye using the MMIP, followed by application of a photoelectrocatalytic treatment. The findings demonstrate an alternative application of this selective material, additional to its use in solid phase extraction or as a sensor platform.

\section{Materials and methods}

\section{Reagents and materials}

Disperse red 73 was obtained from Classic Dyestuffs Inc. (USA). Deionized water was from a Milli-Q system (Millipore).

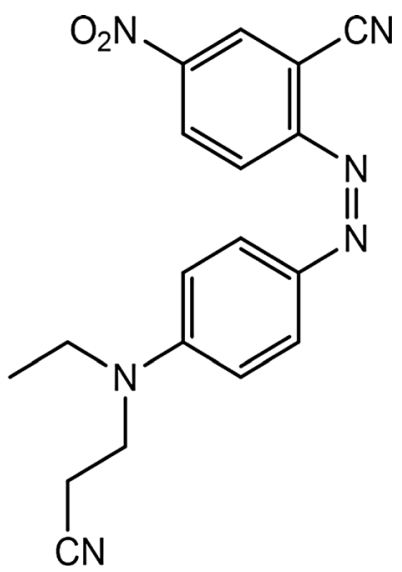

Fig. 1 Structure of disperse red 73 dye (DR73) 
Acetonitrile, acetone, acetic acid, methanol, acrylonitrile (functional monomer), ethylene glycol dimethacrylate (EGDMA), and 2,2-azoisobutyronitrile (AIBN) were purchased from VETEC. The reagents were of analytical grade.

The reagents used for the synthesis of the magnetic nanoparticles were as follows: iron (II) chloride tetrahydrate $\left(\mathrm{FeCl}_{2} \cdot 4 \mathrm{H}_{2} \mathrm{O}\right)$, iron (III) chloride hexahydrate $\left(\mathrm{FeCl}_{3} \cdot 6 \mathrm{H}_{2} \mathrm{O}\right)$, $\mathrm{NH}_{4} \mathrm{OH}$, tetraethyl orthosilicate (TEOS), methacry loxypropyltrimethoxysilane (MPS), ethanol, and toluene (all purchased from Sigma-Aldrich).

Stock solutions of Disperse Red $73\left(10^{-8}\right.$ to $\left.10^{-3} \mathrm{~mol} \mathrm{~L}^{-1}\right)$ were obtained by dissolving the solid standard in methanol. The solutions were stored at $-20^{\circ} \mathrm{C}$.

\section{Instrumentation}

The spectrophotometric measurements were carried out using a TECAN Sunrise microplate reader and Magellan v. 4.0 software. Scanning electron microscopy (SEM) images were obtained with a Hitachi S-570 instrument. Transmission electron microscopy (TEM) images were obtained with a JEOL JEM-2011 instrument equipped with an EDS detector (Oxford Instruments). The microscope was operated at $200 \mathrm{kV}$, with an acceleration voltage of $80-200 \mathrm{kV}$.

\section{Computational simulation}

The interaction between the monomer and the template is one of the critical factors for satisfactory synthesis of a highly selective MIP. The simulations therefore focused on obtaining information on the affinity between dyes and various commercially available monomers that are commonly employed in the synthesis of MIPs for different analytes.

The computer simulations for selection of the best monomer were performed using the HyperChem 8.0.5 program (Hypercube, Inc., Gainesville, Florida, USA) to generate and minimize the molecules. The procedures were performed with the OpenEye software package (under academic license, OpenEye Scientific Software Inc., Santa Fe, New Mexico, USA). The entire process was scripted, automated, and executed using Autolt 3.3.6.0, which uses scripts to automate repetitive processes during the modeling, and MMH with Mopac2009, which is a utility that uses an approach employing semi-empirical quantum chemical calculations. The parameters chosen in the MMH program were the binding energy values of hydrogen bonds and aromatic, hydrophobic, electrostatic, van der Waals, and dipole-dipole interactions. Twenty monomers labeled MP1 to MP20 were evaluated (Table 1).
Table 1 Monomers commonly used in MIP synthesis and chosen for performing computational simulations

\begin{tabular}{ll}
\hline Symbol & Monomer \\
\hline MP1 & $N, N^{\prime}$-methylenebisacrylamide \\
MP2 & Imidazole-4-acrylic acid \\
MP3 & Imidazole-4-acrylic ethylester \\
MP4 & Acrylic acid (AA) \\
MP5 & Acrylamide (ACL) \\
MP6 & Acrolein \\
MP7 & Allylamine \\
MP8 & Acrylonitrile \\
MP9 & Ethylene glycol dimethacrylate \\
MP10 & 2-(cyanoethylamino)ethylmethacrylate \\
MP11 & Methylene succinic acid \\
MP12 & Methacrylic acid \\
MP13 & 3-divinylbenzene \\
MP14 & 4-divinylbenzene \\
MP15 & Styrene \\
MP16 & 1-vinylimidazole \\
MP17 & 2-vinylpyridine \\
MP18 & 4-vinylpyridine \\
MP19 & 2-acrylamido-2-methyl-1-propanesulfonic acid \\
MP20 & 2-hydroxyethyl methacrylate \\
\hline & \\
\hline
\end{tabular}

\section{Preparation of MNPs}

Magnetic nanoparticles (MNPs) were prepared by the precipitation method involving the mixing of $\mathrm{FeCl}_{2} \cdot 4 \mathrm{H}_{2} \mathrm{O}(1.72 \mathrm{~g}$ ) and $\mathrm{FeCl}_{3} \cdot 6 \mathrm{H}_{2} \mathrm{O}(4.72 \mathrm{~g})$, which were dissolved in $80 \mathrm{~mL}$ of water with vigorous stirring under anaerobic conditions (using nitrogen). A $10-\mathrm{mL}$ volume of $\mathrm{NH}_{4} \mathrm{OH}$ (28 wt.\%) was added to the system dropwise, and the reaction medium (at $\mathrm{pH}$ 9-10) was maintained at $80^{\circ} \mathrm{C}$ for $30 \mathrm{~min}$. The black solid formed $\left(\mathrm{Fe}_{3} \mathrm{O}_{4}\right)$ was separated with a $\mathrm{Nd}$ magnet and washed with water to remove the unreacted chemicals, followed by drying under vacuum. The magnetic $\mathrm{Fe}_{3} \mathrm{O}_{4}$ nanoparticles were modified further by dispersing $300 \mathrm{mg}$ in $40 \mathrm{~mL}$ of ethanol and $4 \mathrm{~mL}$ of ultrapure water, with ultrasonication for $15 \mathrm{~min}$, followed by the addition of $5.0 \mathrm{~mL}$ of $\mathrm{NH}_{4} \mathrm{OH}$ and $2.0 \mathrm{~mL}$ of tetraethoxysilane (TEOS), resulting in formation of the $\mathrm{Fe}_{3} \mathrm{O}_{4} @ \mathrm{SiO}_{2}$ material. The mixture was left to react for $12 \mathrm{~h}$ at room temperature, with stirring. The product was collected by magnetic separation, washed with ultrapure water, and dried under vacuum. In the next step, the $\mathrm{Fe}_{3} \mathrm{O}_{4} @ \mathrm{SiO}_{2}$ was modified with 3-methacryloxypropyltrimethoxysilane (MPS) by dispersing $250 \mathrm{mg}$ of the $\mathrm{Fe}_{3} \mathrm{O}_{4} @ \mathrm{SiO}_{2}$ in $50 \mathrm{~mL}$ of anhydrous toluene containing $5 \mathrm{~mL}$ of MPS. The mixture was allowed to react for $12 \mathrm{~h}$, under nitrogen bubbling, giving rise to the $\mathrm{Fe}_{3} \mathrm{O}_{4} @ \mathrm{SiO}_{2}-\mathrm{C}=\mathrm{C}$ material (Valero-Navarro et al. 2011; Hussain et al. 2016; Uzuriaga-Sánchez et al. 2016). 
Following magnetic separation, the solid matter was washed with ultrapure water and dried under vacuum.

\section{Preparation of MMIPs and MNIPs}

MMIPs were prepared by the precipitation-polymerization method, using $0.8 \mathrm{mmol}$ of functional monomer dissolved in ethanol $(30.0 \mathrm{~mL})$ and $0.2 \mathrm{mmol}$ of textile dye (DR73). The mixture was shaken at $25{ }^{\circ} \mathrm{C}$ for $12 \mathrm{~h}$ to enable interaction between the reagents. Afterward, $200 \mathrm{mg}$ of $\mathrm{Fe}_{3} \mathrm{O}_{4} @ \mathrm{SiO}_{2}-$ $\mathrm{C}=\mathrm{C}$ was added to the system, with shaking for a further $3 \mathrm{~h}$, followed by addition of $4.0 \mathrm{mmol}$ of EGDMA and $0.05 \mathrm{mmol}$ of AIBN, with sonication for $5 \mathrm{~min}$. The reaction medium was then kept at $60{ }^{\circ} \mathrm{C}$ for $24 \mathrm{~h}$ under a flow of nitrogen. After polymerization, the template molecule was removed by Soxhlet extraction using methanol/acetic acid $(9: 1, v / v)$ as eluent, which was replaced every $12 \mathrm{~h}$ for 4 days. The MNIPs were prepared under the same conditions as the MIPs but without addition of the DR73 dye. A schematic diagram of the preparation procedure is provided in Scheme 1 .

\section{Binding and release of DR73 using the magnetic polymers}

The performance of the synthesized MIPs was evaluated using adsorption experiments and the construction of isotherms (Uzuriaga-Sánchez et al. 2016). To this end, 40-mg portions of MMIP or MNIP were weighed out, separately, in 15-mL tubes, and conditioned by adding $4 \mathrm{~mL}$ of methanol and stirring for $3 \mathrm{~h}$. The supernatant was then separated from the particles with the aid of a $\mathrm{Nd}$ magnet, followed by the addition of $4.0 \mathrm{~mL}$ of methanol to each tube containing the MMIPs and MNIPs, with stirring for a further $2 \mathrm{~h}$ in order to obtain homogeneous suspensions containing $10 \mathrm{mg} \mathrm{mL}^{-1}$ of the magnetic polymers. Seven different solutions of DR73 in

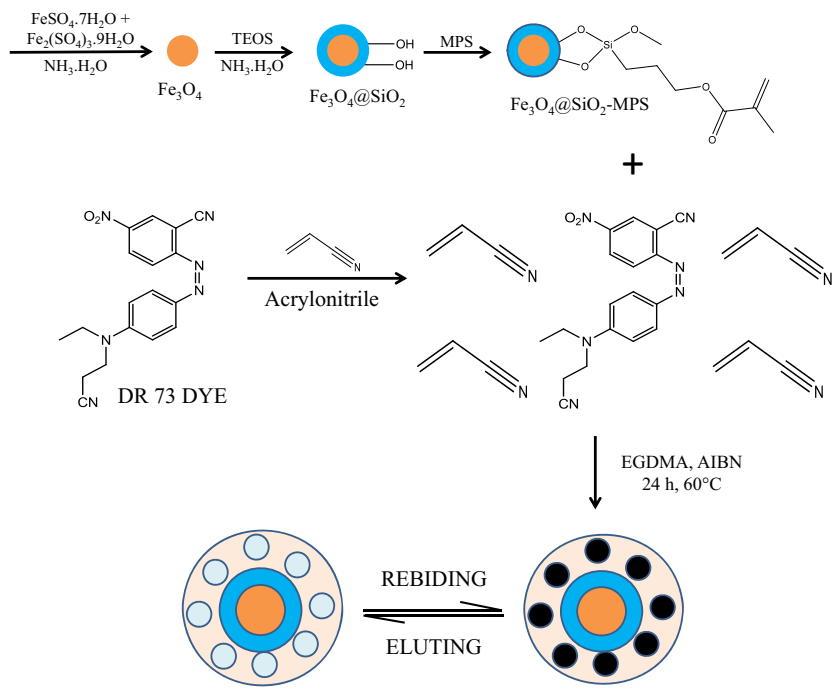

Scheme 1 The representation of the synthetic strategy for DR73 MMIPs by the precipitation method of polymerization

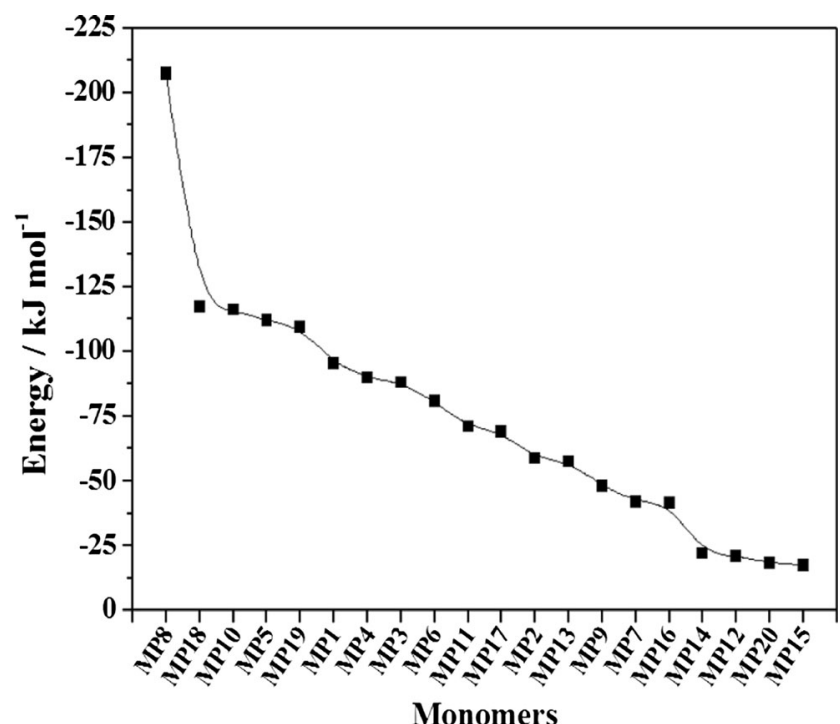

Fig. 2 Results of simulations to evaluate the energies of interaction between DR73 and the 20 monomers listed in Table 1

methanol were then prepared at concentrations between $1.0 \times 10^{-8}$ and $1.0 \times 10^{-2} \mathrm{~mol} \mathrm{~L}^{-1}$, and $500 \mu \mathrm{L}$ of each solution was placed separately in Eppendorf tubes containing $200 \mu \mathrm{L}$ of the MMIP or MNIP suspensions, followed by rotary shaking for $2 \mathrm{~h}$ at room temperature. Subsequently, the supernatant was separated from the magnetic portion of each solution and the unbound dye concentration was measured by UV-Vis spectrophotometry at $509 \mathrm{~nm}$. Comparison was made of the absorbance values obtained in the experiments using MMIPs and MNIPs.

The release of the dye from the MMIPs or MNIPs was studied by extracting the analyte with methanol/acetic acid $(9: 1, v / v)$. Following the extraction, the supernatant was separated from the magnetic portion of each solution and the absorbance was measured by UV-Vis spectrophotometry at $509 \mathrm{~nm}$.

\section{Preparation and characterization of $\mathrm{TiO}_{2} \mathrm{NTs}$ photoanodes used in photoelectrocatalytic experiments}

Titanium plates (Realum, Brazil) sized $3 \times 5 \mathrm{~cm}^{2}$ were polished using silicon carbide sandpaper of successively finer roughness, followed by cleaning with acetone, isopropanol, and finally ultrapure water. $\mathrm{TiO}_{2}$ nanotubes $\left(\mathrm{TiO}_{2} \mathrm{NTs}\right)$ were grown for $50 \mathrm{~h}$ by electrochemical anodization at $30 \mathrm{~V}$, using a Minipa MPL-1303 power supply, with a $25-\mathrm{cm}^{2}$ DSA $®$ (Denora) sheet as a counter electrode, in an organic electrolyte consisting of $0.25 \%$ ammonium fluoride (98\%, Synth) in glycerol (99.5\%, Synth) containing $10 \%(v / v)$ Milli-Q water. Subsequently, the samples were rinsed with deionized water, dried in a nitrogen stream, and annealed at $450^{\circ} \mathrm{C}$ for $30 \mathrm{~min}$ in air, using a heating rate of $2^{\circ} \mathrm{C} \mathrm{min}^{-1}$ (Bessegato et al. 2016).

The $\mathrm{TiO}_{2} \mathrm{NTs}$ electrode was characterized by X-ray diffraction (XRD), using a Siemens D5000 diffractometer operated 
Fig. 3 TEM images of a DR73MMIP and b MNIP (at $\times 30,000$ magnification)
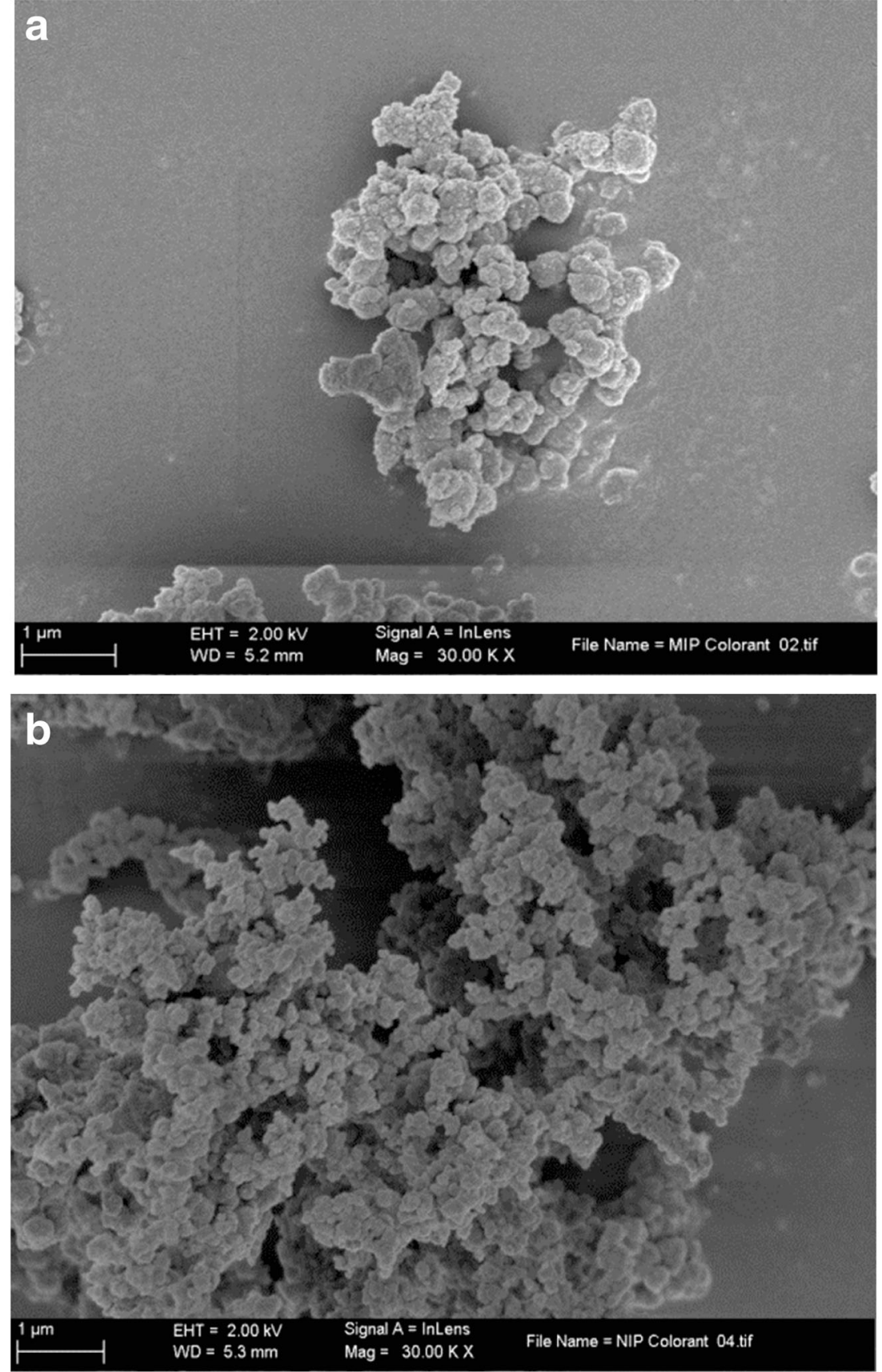

with $\mathrm{Cu} \mathrm{K} \alpha$ radiation and controlled with Diffrac Plus XRD Commander software. Morphological characterization was performed by field emission gun-scanning electron microscopy (FEG-SEM), using a JEOL 7500F instrument.

\section{Photoelectrocatalytic degradation of DR73 released from the magnetic polymers}

Photoelectrocatalytic degradation of DR73 released from the MMIPs was performed after adsorption of the dye from wastewater samples spiked with $1.0 \times 10^{-5}$ and $1.0 \times 10^{-4} \mathrm{~mol} \mathrm{~L}^{-1}$ of DR73 and subsequent elution with methanol/water $(1: 1$, $v / v)$. A lower proportion of methanol was used in this procedure, due to its competition with DR73 for the $\cdot \mathrm{OH}$ radicals responsible for the degradation of organic compounds.

The dye released was collected and subjected to photoelectrocatalysis treatment. The degradations were performed in a $100-\mathrm{mL}$ cylindrical glass reactor at a temperature controlled at $20{ }^{\circ} \mathrm{C}$ using a thermostatic bath. The $\mathrm{TiO}_{2} \mathrm{NTs}$ photoelectrode was irradiated using a commercial highpressure $\mathrm{Hg}$ lamp $(125 \mathrm{~W})$ that was inserted vertically in a central quartz tube. The reactor consisted of a two-electrode cell in which a DSA® plate was used as the cathode and the $\mathrm{TiO}_{2} \mathrm{NTs}$ electrode as the anode. The electrolyte was $0.1 \mathrm{M}$ 
$\mathrm{Na}_{2} \mathrm{SO}_{4}$ and all the photoelectrochemical experiments were carried out using a power supply operated at a constant voltage of $2.5 \mathrm{~V}$. Aliquots were removed at specified times during the DR73 degradation for analysis by liquid chromatographymass spectrometry (LC-MS/MS).

\section{LC-MS/MS}

The DR73 concentration was determined using a liquid chromatograph (Model 1200, Agilent Technologies) coupled to a Model 3200 QTRAP quadrupole/linear ion trap mass spectrometer (AB SCIEX Instruments) operated in positive mode with Turbo Ion Spray ionization.

The chromatographic separation was performed using a Luna C-18 column $(250 \mathrm{~mm} \times 4.6 \mathrm{~mm}, 5 \mu \mathrm{m}$ particle size $)$ and a mobile phase consisting of a mixture of water and acetonitrile $(20: 80, v / v)$, both containing $0.1 \%$ formic acid, in isocratic mode at $1 \mathrm{~mL} \mathrm{~min}^{-1}$. The injection volume and column temperature were $10 \mu \mathrm{L}$ and $25^{\circ} \mathrm{C}$, respectively. The analysis time was $10 \mathrm{~min}$. The mass spectrometric detection was performed using the Model 3200 QTRAP linear ion trap analyzer in enhanced mass scan (EMS) mode, with prior optimization of the ionization conditions and source parameters by direct infusion of a DR73 standard at a concentration of $500 \mu \mathrm{g} \mathrm{L}^{-1}$ in methanol/water $(1: 1, v / v$, containing $0.1 \%$ formic acid) using an automated syringe at a flow rate of $10 \mu \mathrm{L} \mathrm{min}{ }^{-1}$. Ionization was achieved with a Turbo Ion Spray electrospray source operated in positive mode. The source parameters were as follows: $5500 \mathrm{~V} ; 550{ }^{\circ} \mathrm{C}$; Gas 1 : 50 psi; Gas 2: 50 psi; curtain gas: 15 psi. The ionization conditions were optimized for DR73, with declustering potential (DP) and entrance potential (EP) values of 36 and $8.0 \mathrm{~V}$, respectively. Fragment ion analyses were performed using the linear ion trap in enhanced product ion (EPI) mode, in order to obtain structural information about the products formed. The collision energy used in these experiments was in the range from 20 to $50 \mathrm{~V}$.

\section{Results and discussion}

\section{Computational simulation}

The results of the computational simulation provided information on the interaction of the DR73 dye with 20 commercial monomers often employed for the synthesis of MIPs (Table 1). The binding energies of the interactions are illustrated in Fig. 2. The M8 monomer (acrylonitrile) was most suitable for synthesis of the MIP for DR73, due to its high free energy, which provided better interaction with the analyte. Hence, this monomer was selected for use in the synthesis.

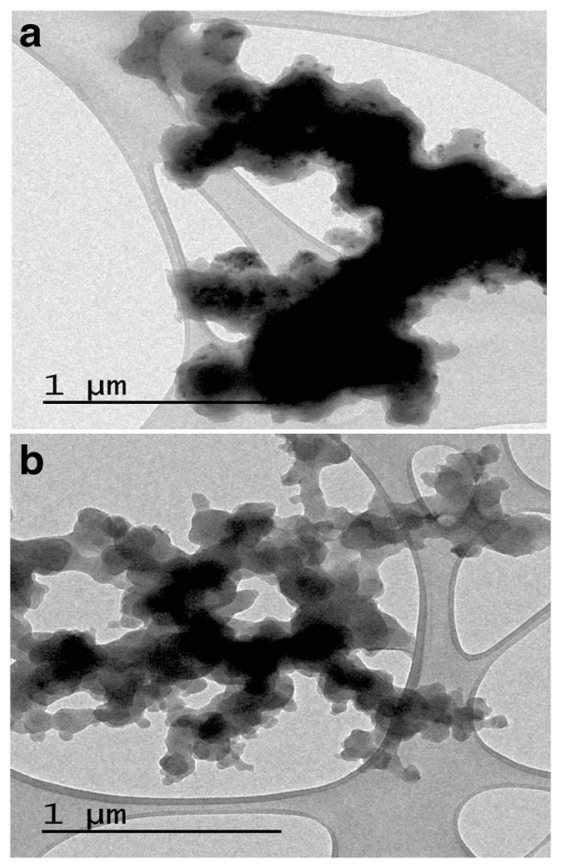

Fig. 4 TEM images of a DR73-MMIP and b MNIP (at $\times 5000$ magnification)

\section{Characterization of the DR73-MMIP}

Morphological characterization of the prepared DR73-MMIP and MNIP materials was performed using scanning and transmission electron microscopy (SEM and TEM). Figures 3 and 4 show the micrographs obtained for the acrylonitrile-based MNIP and DR73-MIP samples, respectively.

The particles shown in Fig. 3 were porous and homogeneous, exhibiting the "cauliflower clusters" appearance characteristic of other magnetic MIPs reported in the literature (Hussain et al. 2016; Uzuriaga-Sánchez et al. 2016). It can be seen from Fig. 3a that the DR73-MMIP particles presented greater agglomeration, compared to MNIP, which could be attributed to a greater content of magnetic nanoparticles, as shown by the TEM micrographs (Fig. 4). Another interesting observation was the substantial polymeric coating formed on the outer shell enclosing $\mathrm{Fe}_{3} \mathrm{O}_{4}$ at the core (Fig. 4), resulting in an MMIP with excellent magnetite encapsulation.

\section{Spectrophotometric analysis of binding between the dye and the magnetic MIPs}

The adsorption isotherm describes the amount of a particular solute adsorbed by an adsorbent surface (Q), as a function of the equilibrium solute concentration. In order to construct an adsorption isotherm, the adsorbent (DR73-MMIP or MNIP) should be placed in contact with the solution containing the component to be adsorbed (in this case DR73) at varying 


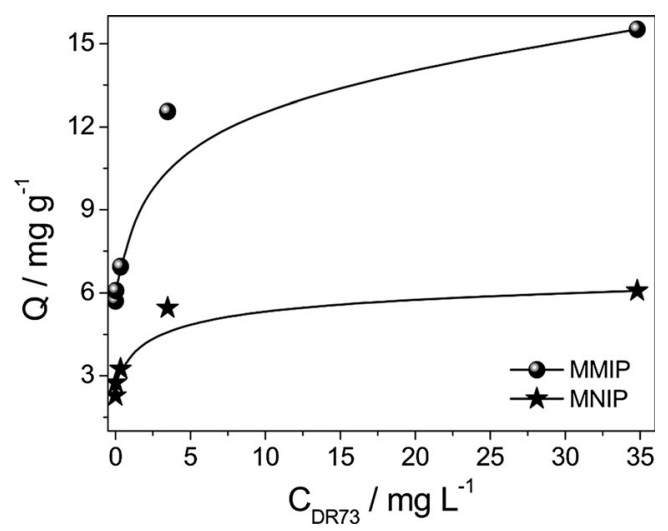

Fig. 5 Isotherms for the adsorption of DR73 by MMIP (black circle) and MNIP (black star). Experimental conditions: V $=700 \mu \mathrm{L} ; 2 \mathrm{mg}$ of polymer; $2 \mathrm{~h}$ of equilibrium time

concentrations and at constant temperature, until equilibrium is attained. The amount of adsorbed material can then be determined using a suitable analytical methodology (Tagliaferro et al. 2011). The value of $Q$ for the construction of isotherms is estimated by Eq. 1 (Wong et al. 2016):

$Q=\frac{\left(C_{i}-C_{f}\right) V}{m_{p o l}}$,

where $V(\mathrm{~L})$ is the total volume (here $700 \mu \mathrm{L}$ ) and $m_{p o l}$ is the mass ( $\mathrm{g}$ ) of the adsorbent polymer (MMIP or MNIP) in the mixture.
The amounts of dye bound to the polymers were calculated using the supernatant absorbance values (at $509 \mathrm{~nm}$ ), subtracting the amount of unbound dye $\left(\mathrm{C}_{\mathrm{f}}\right.$, in $\left.\mathrm{mg} \mathrm{L}^{-1}\right)$ from the initial amount $\left(C_{i}\right.$, in $\left.\mathrm{mg} \mathrm{L}^{-1}\right)$ of dye added to the mixture (Scheme 2). Figure 5 shows the isotherms for analyte adsorption by the DR73-MMIP and MNIP materials.

A much larger amount of dye was bound to MMIP $\left(\mathrm{Q}_{\max } \rightarrow 16 \mathrm{mg} \mathrm{g}^{-1}\right)$, compared to MNIP ( $\mathrm{Q}_{\max } \rightarrow$ $6 \mathrm{mg} \mathrm{g}^{-1}$ ), indicating that the cavities formed in the MIP led to a higher probability of analyte insertion in these specific cavities by selective recognition, resulting in a 2.7-fold higher retention by the MMIP adsorbent.

Scheme 2 illustrates the binding procedure for dye extraction by MMIP in $2 \mathrm{~h}$, with lower absorbance for MMIP, compared to MNIP, due to insertion of the dye in the MMIP cavities created by the template. This did not occur in the case of MNIP, with the dye molecules remaining dispersed in solution, causing a greater absorbance of the final dye solution $\left(\mathrm{Abs}_{\mathrm{f}}\right.$ IN MNIP in Scheme 2), compared to the absorbance of the final solution for MMIP $\left(\mathrm{Abs}_{\mathrm{f}} \mathrm{IN}\right.$ MMIP).

\section{Spectrophotometric analysis of dye release from the magnetic MIPs}

Table 2 shows the absorbance values corresponding to the dye released from the MMIP and MNIP. The results confirmed the substantial difference between MMIP and MNIP in terms
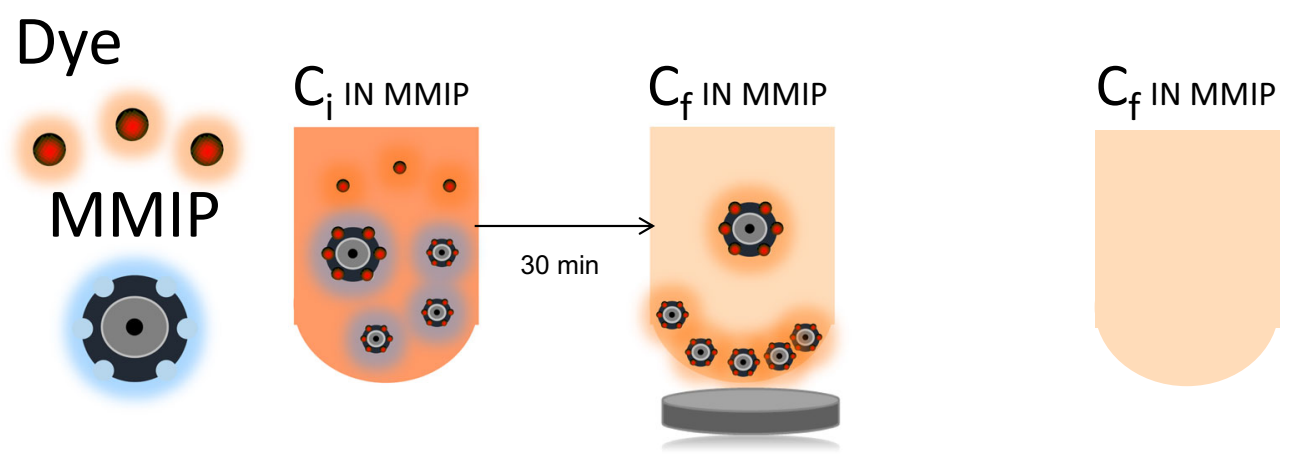

$C_{i}$ IN MMIP $=C_{i}$ IN MNIP

$\mathrm{AbS}_{\mathrm{f}}$ IN MMIP $<\mathrm{AbS}_{\mathrm{f}}$ IN MNIP
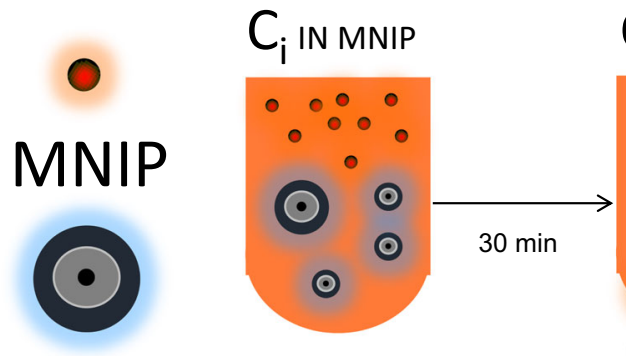

$C_{f}$ IN MNIP

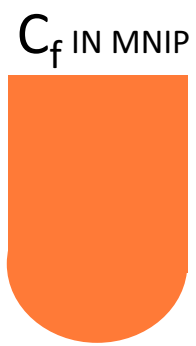

Scheme 2 Illustration of dye binding in the MMIP and MNIP after $2 \mathrm{~h}$ and analysis of the supernatants by UV-Vis spectroscopy at $509 \mathrm{~nm}$ 
Table 2. Comparison of the percentages of dye released from the MMIP and MNIP after extraction with methanol and acetic acid

\begin{tabular}{|c|c|c|c|c|c|c|}
\hline \multirow{3}{*}{$\begin{array}{l}\text { Dye } \\
\text { concentration } \\
\left(\mathrm{mg} \mathrm{L}^{-1}\right)\end{array}$} & \multicolumn{6}{|c|}{ Absorbance of the supernatant after extraction } \\
\hline & \multicolumn{3}{|l|}{ MMIP } & \multicolumn{3}{|l|}{ MNIP } \\
\hline & Expected & Obtained & $\begin{array}{l}\text { Percentage of } \\
\text { DR73 released } \\
\text { from MMIP }(\%)\end{array}$ & Expected & Obtained & $\begin{array}{l}\text { Percentage of } \\
\text { DR73 released } \\
\text { from MNIP (\%) }\end{array}$ \\
\hline 0.00348 & 0.292 & 0.201 & 68.67 & 0.117 & 0.117 & 100.0 \\
\hline 0.0348 & 0.313 & 0.213 & 68.13 & 0.141 & 0.137 & 97.16 \\
\hline 0.348 & 0.355 & 0.234 & 66.04 & 0.167 & 0.145 & 86.82 \\
\hline 3.48 & 0.644 & 0.340 & 52.79 & 0.280 & 0.211 & 75.36 \\
\hline 34.8 & 0.795 & 0.408 & 51.36 & 0.312 & 0.228 & 73.08 \\
\hline
\end{tabular}

of the dye adsorption mechanism. Interestingly, there was $100 \%$ dye removal from MNIP, which could be explained by the non-specific interactions between the polymer and the dye on the surface of the MNIP, rather than within selective cavities. Hence, it was easier to remove the dye from this adsorbent (MNIP), but the material was consequently less suitable for the treatment of wastewater containing the analyte. Scheme 3 illustrates the release procedure used for dye extraction from MMIP in $2 \mathrm{~h}$.

\section{Photoelectrocatalytic oxidation of disperse red 73 dye following extraction from wastewater using the MMIP}

Given that the MMIP was able to efficiently extract the DR73 dye from a methanol/water $(1: 1, v / v)$ mixture, the next step was to evaluate the extraction from an aqueous solution in which the analyte was likely to be present. The method was applied using wastewater samples spiked with the dye at concentrations at which the greatest difference in adsorption between the MMIP and MNIP was obtained, namely,
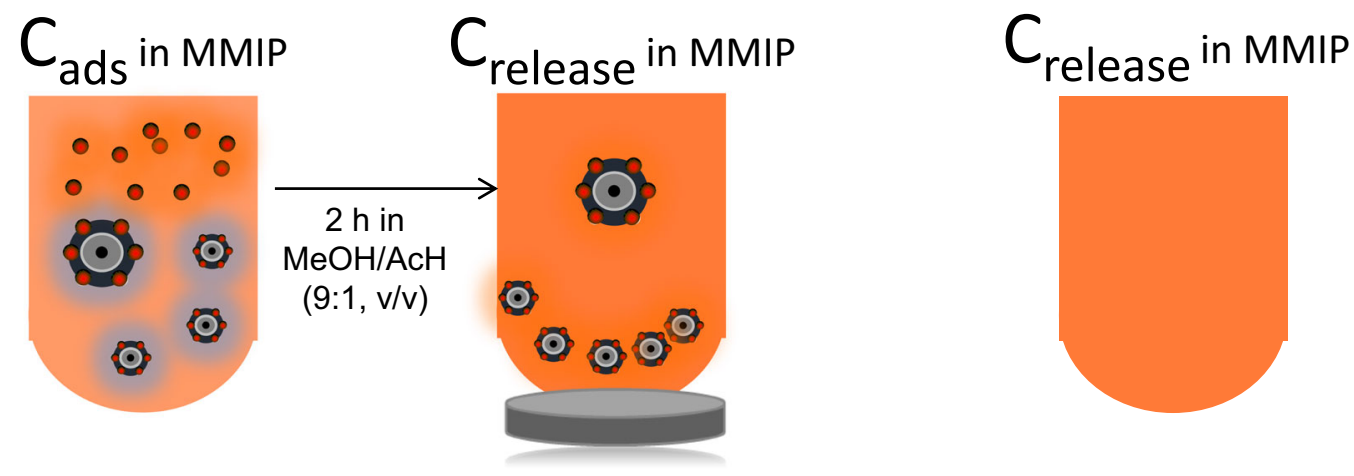

\section{$A b s_{\text {rel MMIP }}>A b s_{\text {rel MNIP }}$}
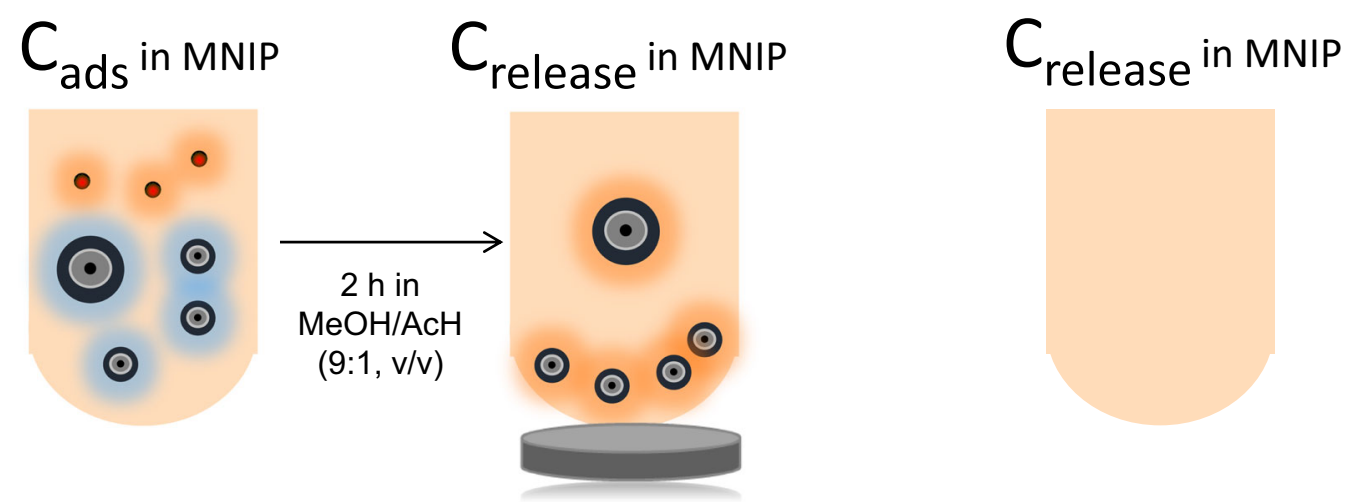

Scheme 3 Illustration of dye release (rel) from the MMIP and MNIP after extraction with methanol:acetic acid (1:9, v/v) and analysis of the supernatants by UV-Vis spectroscopy at $509 \mathrm{~nm}$ 

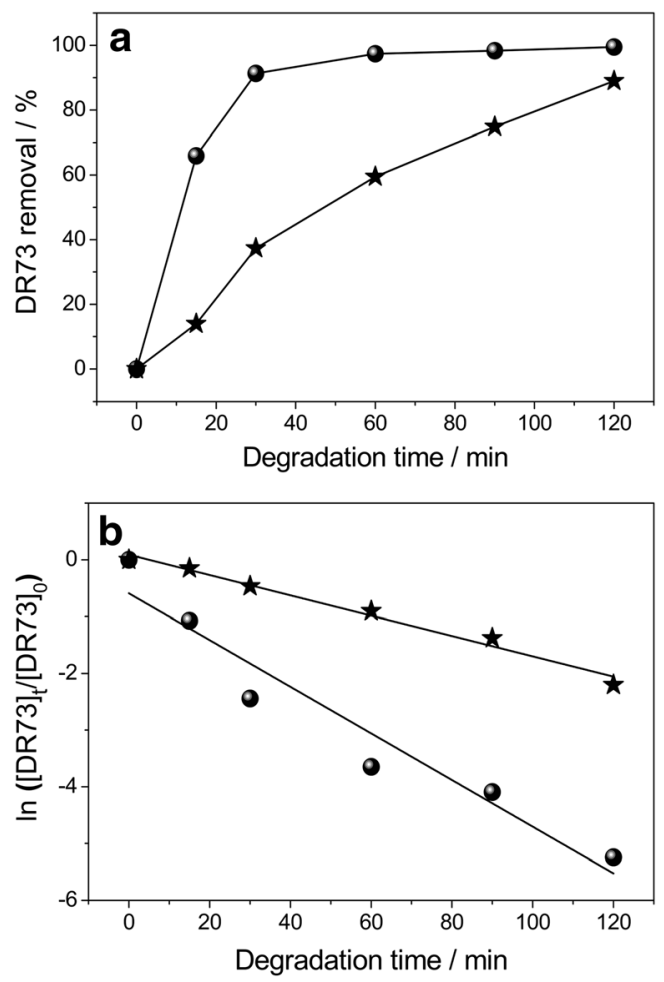

Fig. 6 a DR73 removal vs. degradation time during the photoelectrocatalytic oxidation; b pseudo-first-order kinetic curves constructed using initial concentrations of (black star) $2.29 \times 10^{-5} \mathrm{~mol} \mathrm{~L}^{-1}$ and (black circle) $8.42 \times 10^{-6} \mathrm{~mol} \mathrm{~L}^{-1}$

$1.0 \times 10^{-5} \mathrm{~mol} \mathrm{~L}^{-1}\left(3.48 \mathrm{mg} \mathrm{L}^{-1}\right)$ and $1.0 \times 10^{-4} \mathrm{~mol} \mathrm{~L}^{-1}$ (34.8 $\mathrm{mg} \mathrm{L}^{-1}$ ). The conditions were the same as those adopted throughout this investigation $(2 \mathrm{~h}$ for adsorption and $2 \mathrm{mg}$ of adsorbent). Afterward, a Nd magnet was used to separate the DR73-MMIP, and the dye solution collected was analyzed. The dye concentrations in the supernatants were $8.42 \times 10^{-6}$ and $2.29 \times 10^{-5} \mathrm{~mol} \mathrm{~L}^{-1}$ for the two starting solutions, respectively, corresponding to recoveries of 84.2 and $22.9 \%$. These findings indicated that the MMIP could be an effective tool for use in the removal of dye from dilute effluents.

The photoelectrocatalytic treatment efficiency was evaluated by transferring the eluted dye to a one-compartment photoelectrocatalytic reactor containing $\mathrm{Ti} / \mathrm{TiO}_{2}$ nanotubes as the photoanode. The sample was then treated by applying a bias potential of $2.5 \mathrm{~V}$ and UV/Vis irradiation for $120 \mathrm{~min}$. Aliquots were collected during the photoelectrocatalytic treatment for analysis by HPLC/MS/MS. The resulting dye removal percentages are shown in Fig. 6a. Mass spectra were obtained for aliquots of both extracts (containing $8.42 \times 10^{-6}$ and $2.29 \times 10^{-5} \mathrm{~mol} \mathrm{~L}^{-1}$ of dye) during the course of the photoelectrocatalytic degradation. Surprisingly, it was not possible to detect intermediates produced during the treatment (see Table S1). However, there were significant decreases in the dye concentrations during the treatments. The dye retention times obtained before and after degradation were around $4.40 \mathrm{~min}$ for both concentrations studied. Both samples showed almost complete dye removal after $120 \mathrm{~min}$ of treatment.

The degradation followed pseudo-first-order kinetics (Langmuir-Hinshelwood kinetics) (Fig. 6b), with degradation rate constants of -0.018 and $-0.041 \mathrm{~min}^{-1}$ for the starting concentrations of $2.29 \times 10^{-5}$ and $8.42 \times 10^{-6} \mathrm{~mol} \mathrm{~L}^{-1}$, respectively. As expected, a higher initial concentration resulted in a lower rate constant and a longer time required for $90 \%$ removal. The results demonstrated the viability of DR73 removal by adsorption onto a selective material followed by photoelectrocatalytic degradation. The findings open up new perspectives for the decontamination of polluted water, enabling a harmful contaminant to be removed from a large volume of aqueous medium using MMIPs, followed by transfer of the contaminant to another medium in which it can be completely destroyed using an advanced oxidation process.

\section{Conclusions}

This work shows the potential of using magnetic molecularly imprinted polymers for the efficient removal of contaminants from complex wastewater samples, extending the applications of MIPs beyond their use in SPE extraction or in sensors. The use of these selective and reusable polymers, allied with degradation techniques such as the one employed here, could assist in the remediation or decontamination of effluents, contributing toward a responsible approach to environmental protection.

Acknowledgements The authors are grateful for the financial support provided by the Brazilian agencies Conselho Nacional de Desenvolvimento Científico e Tecnológico (CNPq, no. 200309/2015-3 and no. 400459/2012-4) and Fundação de Amparo à Pesquisa do Estado de São Paulo (FAPESP, no. 2014/25264-3 and no. 2014/03679-7) and the Spanish Ministry of Economy and Competitiveness (MINECO, no. BIO2013-41242-R).

\section{References}

Ahmaruzzaman M (2011) Industrial wastes as low-cost potential adsorbents for the treatment of wastewater laden with heavy metals. Adv Colloid Interfac 166:36-59. doi:10.1016/j.cis.2011.04.005

Arslan İ, Akmehmet Balcioğlu I, Tuhkanen T (1999) Oxidative treatment of simulated dyehouse effluent by uv and near-UV light assisted Fenton's reagent. Chemosphere 39:2767-2783. doi:10.1016 /S0045-6535(99)00211-8

Bessegato GG, Guaraldo TT, de Brito JF et al (2015) Achievements and trends in photoelectrocatalysis: from environmental to energy applications. Electrocatalysis 6:415-441. doi:10.1007/s12678-015-0259-9

Bessegato GG, Cardoso JC, da Silva BF, Zanoni MVB (2016) Combination of photoelectrocatalysis and ozonation: a novel and powerful approach applied in acid yellow 1 mineralization. Appl Catal B-Environ 180:161-168. doi:10.1016/j.apcatb.2015.06.013 
Cardoso JC, Lucchiari N, Zanoni MVB (2015) Bubble annular photoeletrocatalytic reactor with $\mathrm{TiO}_{2}$ nanotubes arrays applied in the textile wastewater. J Environ Chem Eng 3:1177-1184. doi:10.1016/j.jece.2015.04.010

Carneiro PA, Oliveira DP, Umbuzeiro GA, Zanoni MVB (2009) Mutagenic activity removal of selected disperse dye by photoelectrocatalytic treatment. J Appl Electrochem 40:485-492. doi:10.1007/s10800-009-0018-9

Dirion B, Cobb Z, Schillinger E et al (2003) Water-compatible molecularly imprinted polymers obtained via high-throughput synthesis and experimental design. J Am Chem Soc 125:15101-15109. doi: $10.1021 / \mathrm{ja} 0355473$

Ghaly AE, Ananthashankar R, Alhattab M, Ramakrishnan VV (2014) Production, characterization and treatment of textile effluents: a critical review. J Chem Eng Process Technol 5:1-18. doi:10.4172 /2157-7048.1000182

Grimes CA, Mor GK (2009) $\mathrm{TiO}_{2}$ nanotube arrays: synthesis, properties, and applications. Springer, New York

Huang D-L, Wang R-Z, Liu Y-G et al (2014) Application of molecularly imprinted polymers in wastewater treatment: a review. Environ Sci Pollut R 22:963-977. doi:10.1007/s11356-014-3599-8

Hussain S, Khan S, Gul S et al (2016) A novel core@shell magnetic molecular imprinted nanoparticles for selective determination of folic acid in different food samples. React Funct Polym 106:5156. doi:10.1016/j.reactfunctpolym.2016.07.011

Jiang M, Zhang J, Mei S et al (2006) Direct enrichment and high performance liquid chromatography analysis of ultra-trace bisphenol a in water samples with narrowly dispersible bisphenol a imprinted polymeric microspheres column. J Chromatogr A 1110:27-34. doi:10.1016/j.chroma.2006.01.051

Jin XC, Liu GQ, Xu ZH, Tao WY (2007) Decolorization of a dye industry effluent by Aspergillus fumigatus XC6. Appl Microbiol Biot 74: 239-243. doi:10.1007/s00253-006-0658-1

Lavignac N, Allender CJ, Brain KR (2004) Current status of molecularly imprinted polymers as alternatives to antibodies in sorbent assays. Anal Chim Acta 510:139-145. doi:10.1016/j.aca.2003.12.066

Liu N, Paramasivam I, Yang M, Schmuki P (2012) Some critical factors for photocatalysis on self-organized $\mathrm{TiO}_{2}$ nanotubes. J Solid State Electrochem 16:3499-3504. doi:10.1007/s10008-012-1799-z

Murray A, Örmeci B (2012) Application of molecularly imprinted and non-imprinted polymers for removal of emerging contaminants in water and wastewater treatment: a review. Environ Sci Pollut R 19: 3820-3830. doi:10.1007/s11356-012-1119-2

Panizza M, Cerisola G (2008) Removal of colour and COD from wastewater containing acid blue 22 by electrochemical oxidation. $\mathrm{J}$ Hazard Mater 153:83-88. doi:10.1016/j.jhazmat.2007.08.023

Paschoal FMM, Anderson MA, Zanoni MVB (2009) The photoelectrocatalytic oxidative treatment of textile wastewater containing disperse dyes. Desalination 249:1350-1355. doi:10.1016/j. desal.2009.06.024

Robinson T, McMullan G, Marchant R, Nigam P (2001) Remediation of dyes in textile effluent: a critical review on current treatment technologies with a proposed alternative. Bioresour Technol 77:247255. doi:10.1016/S0960-8524(00)00080-8

Salleh MAM, Mahmoud DK, Karim WAWA, Idris A (2011) Cationic and anionic dye adsorption by agricultural solid wastes: a comprehensive review. Desalination 280:1-13. doi:10.1016/j. desal.2011.07.019

Sanghi R, Bhattacharya B (2002) Review on decolorisation of aqueous dye solutions by low cost adsorbents. Color Technol 118:256-269. doi:10.1111/j.1478-4408.2002.tb00109.x

Saratale RG, Saratale GD, Chang JS, Govindwar SP (2011) Bacterial decolorization and degradation of azo dyes: a review. J Taiwan Inst Chem E 42:138-157. doi:10.1016/j.jtice.2010.06.006

Shea KJ, Sasaki DY (1991) An analysis of small-molecule binding to functionalized synthetic polymers by $13 \mathrm{C} \mathrm{CP/MAS} \mathrm{NMR} \mathrm{and} \mathrm{FT-}$ IR spectroscopy. J Am Chem Soc 113:4109-4120. doi:10.1021 /ja00011a009

Svenson J, Nicholls IA (2001) On the thermal and chemical stability of molecularly imprinted polymers. Anal Chim Acta 435:19-24. doi:10.1016/S0003-2670(00)01396-9

Tagliaferro GV, Pereira PHF, Rodrigues LÁ, da Silva MLCP (2011) Adsorção de chumbo, cádmio e prata em óxido de nióbio $(\mathrm{V})$ hidratado preparado pelo método da precipitação em solução homogênea. Quim Nov. 34:101-105. doi:10.1590/S010040422011000100020

Tamayo FG, Casillas JL, Martin-Esteban A (2003) Highly selective fenuron-imprinted polymer with a homogeneous binding site distribution prepared by precipitation polymerisation and its application to the clean-up of fenuron in plant samples. Anal Chim Acta 482: 165-173. doi:10.1016/S0003-2670(03)00213-7

Uzuriaga-Sánchez RJ, Khan S, Wong A et al (2016) Magnetically separable polymer (mag-MIP) for selective analysis of biotin in food samples. Food Chem 190:460-467. doi:10.1016/j. foodchem.2015.05.129

Valero-Navarro A, Medina-Castillo AL, Fernandez-Sanchez JF, Fernández-Gutiérrez A (2011) Synthesis of a novel polyurethanebased-magnetic imprinted polymer for the selective optical detection of 1-naphthylamine in drinking water. Biosens Bioelectron 26: 4520-4525. doi:10.1016/j.bios.2011.05.013

Vlatakis G, Andersson LI, Müller R, Mosbach K (1993) Drug assay using antibody mimics made by molecular imprinting. Nature 361:645647. doi:10.1038/361645a0

Wong A, de Oliveira FM, Tarley CRT, Del Pilar Taboada Sotomayor M (2016) Study on the cross-linked molecularly imprinted poly(methacrylic acid) and poly(acrylic acid) towards selective adsorption of diuron. React Funct Polym 100:26-36. doi:10.1016/j. reactfunctpolym.2016.01.006

Wulff G, Sarhan A (1972) The use of polymers with enzyme-analogous structures for the resolution of racemates. Angew Chem Int Ed 11: 341-344

Wulff G, Gross T, Schönfeld R (1997) Enzyme models based on molecularly imprinted polymers with strong esterase activity. Angew Chemie Int Ed English 36:1962-1964. doi:10.1002/anie.199719621

Xie YB, Li XZ (2006) Interactive oxidation of photoelectrocatalysis and electro-Fenton for azo dye degradation using $\mathrm{TiO}_{2}-\mathrm{Ti}$ mesh and reticulated vitreous carbon electrodes. Mater Chem Phys 95:3950. doi:10.1016/j.matchemphys.2005.05.048

Yagub MT, Sen TK, Afroze S, Ang HM (2014) Dye and its removal from aqueous solution by adsorption: a review. Adv Colloid Interfac 209: 172-184. doi:10.1016/j.cis.2014.04.002 